Real-world Effectiveness and Safety of Pazopanib in Patients With Intermediate Prognostic Risk Advanced Renal Cell Carcinoma

\title{
Procopio, Giuseppe
}

2019-06

Procopio , G , Bamias , A , Schmidinger , M , Hawkins , R, Rodriguez Sanchez , A , Vazquez Estevez , S , Srihari , N, Kalofonos , H, Bono , P , Pisal , C B , Hirschberg , Y, Dezzani , L , Ahmad, Q, Suarez Rodriguez , C \& Jonasch , E 2019 , ' Real-world Effectiveness and Safety of Pazopanib in Patients With Intermediate Prognostic Risk Advanced Renal Cell Carcinoma ', Clinical Genitourinary Cancer , vol. 17 , no. 3 , pp. E526-E533 . https://doi.org/10.1016/j.clgc.2019.01.018

http://hdl.handle.net/10138/318789

https://doi.org/10.1016/j.clgc.2019.01.018

publishedVersion

Downloaded from Helda, University of Helsinki institutional repository.

This is an electronic reprint of the original article.

This reprint may differ from the original in pagination and typographic detail.

Please cite the original version. 


\title{
Real-world Effectiveness and Safety of Pazopanib in Patients With Intermediate Prognostic Risk Advanced Renal Cell Carcinoma
}

\author{
Giuseppe Procopio, ${ }^{1}$ Aristotelis Bamias, ${ }^{2}$ Manuela Schmidinger, ${ }^{3}$ Robert Hawkins, ${ }^{4}$ \\ Angel Rodriguez Sánchez, ${ }^{5}$ Sergio Vázquez Estevez, ${ }^{6}$ Narayanan Srihari, ${ }^{7}$ \\ Haralabos Kalofonos, ${ }^{8}$ Petri Bono, ${ }^{9}$ Chaitali Babanrao Pisal, ${ }^{10}$ Yulia Hirschberg, ${ }^{11}$ \\ Luca Dezzani, ${ }^{11}$ Qasim Ahmad, ${ }^{11}$ Cristina Suárez Rodriguez, ${ }^{12}$ Eric Jonasch ${ }^{13}$
}

\section{Abstract}

Patients with intermediate-risk advanced renal cell carcinoma are a heterogeneous population, having either 1 or 2 risk factors. It is unclear whether all patients in this risk category should be treated similarly. A secondary analysis of the PRINCIPAL study of pazopanib found that patients can be stratified by number of risk factors and Eastern Cooperative Oncology Group performance status to more accurately predict outcomes.

Introduction: The objective of this study was to determine the effectiveness and safety of pazopanib in patients with intermediate-risk advanced/metastatic renal cell carcinoma in the PRINCIPAL study (NCT01649778). Patients and Methods: Patients had clear-cell advanced/metastatic renal cell carcinoma and met intermediate-risk International Metastatic Renal Cell Carcinoma Database Consortium (IMDC) and Memorial Sloan Kettering Cancer Center (MSKCC) criteria. Assessments included progression-free survival, overall survival, objective response rate, and safety. We also evaluated effectiveness based on number of risk factors, age, and performance status (PS), as well as safety in older and younger patients. Results: Three hundred sixty three and 343 intermediate-risk MSKCC and IMDC patients were included, respectively. The median progression-free survival was 13.8 months ( $95 \%$ confidence interval [Cl], 10.7-18.1 months) and 7.4 months (95\% Cl, 6.2-10.3 months) for patients with 1 and 2 MSKCC risk factors, respectively, and 13.1 months (95\% Cl, 10.7-18.1 months) and 8.1 months (95\% Cl, 6.4-10.7 months) for patients with 1 and 2 IMDC risk factors, respectively. The median overall survival was not reached and was 15.2 months $(95 \% \mathrm{Cl}, 12.3-26.5$ months) for patients with 1 and 2 MSKCC risk factors, respectively, and 33.9 months ( $95 \% \mathrm{Cl}, 33.9$ months to not estimable) and 19.4 months (95\% $\mathrm{Cl}, 14.3$ months to not estimable) with 1 and 2 IMDC risk factors, respectively. A lower overall response rate was observed with Eastern Cooperative Oncology Group PS $\geq 2$ (vs. PS $<2$ ). All-grade treatment-related adverse events occurred in approximately $63 \%$ of patients, and the safety profile among older and younger patients was similar. Conclusions: Outcomes with pazopanib in intermediate-risk patients suggest that patients can be further stratified by number of risk factors (1 vs. 2) and Eastern Cooperative Oncology Group PS (<2 vs. $\geq 2)$ to more accurately predict outcomes.

Clinical Genitourinary Cancer, Vol. 17, No. 3, e526-33 (c) 2019 Published by Elsevier Inc.

Keywords: Intermediate risk, Pazopanib, Prognosis, Renal cell carcinoma

\footnotetext{
${ }^{1}$ Department of Medical Oncology, Fondazione IRCCS Istituto Nazionale dei Tumori, Milan, Italy

${ }^{2}$ Department of Clinical Therapeutics, National and Kapodistrian University of Athens Alexandra Hospital, Athens, Greece

${ }^{3}$ Department of Medicine, Medical University of Vienna, Vienna, Austria

${ }^{4}$ The Christie NHS Foundation Trust, Christie CRC Research Centre, Manchester, UK

${ }^{5}$ University Hospital of Leon, Campus de Vegazana, León, Spain

${ }^{6}$ Department of Oncology, Hospital Universitario Lucus Augusti, Lugo, Spain

${ }^{7}$ Department of Oncology, Shrewsbury \& Telford Hospitals NHS Trust, Shrewsbury, UK

${ }^{8}$ Division of Oncology, Department of Medicine, University of Patras, Pan-

epistimioupoli Patron, Patra, Greece

${ }^{9}$ Comprehensive Cancer Center, Helsinki University Hospital, Helsinki, Finland
}

\footnotetext{
${ }^{10}$ Novartis Healthcare Private Limited, Salarpuria-Sattva Knowledge City, Raidurg, Hyderabad, India

${ }^{11}$ Novartis Pharmaceuticals Corporation, East Hanover, NJ

${ }^{12}$ Department of Oncology, Vall d'Hebron University Hospital and Institute of

Oncology, Universitat Autònoma de Barcelona, Centro Cellex, Barcelona, Spain

${ }^{13}$ Department of Genitourinary Medical Oncology, The University of Texas MD Anderson Cancer Center, Houston, TX

Submitted: Nov 14, 2018; Revised: Jan 18, 2019; Accepted: Jan 29, 2019

Address for correspondence: Giuseppe Procopio, MD, Department of Medical

Oncology, Fondazione IRCCS Istituto Nazionale dei Tumori, Via Venezian 1, Milan 20133, Italy

E-mail contact: giuseppe.procopio@istitutotumori.mi.it
} 


\section{Introduction}

Clinical outcomes for patients with advanced renal cell carcinoma (RCC) have improved markedly since the introduction of agents targeting vascular endothelial growth factor and its receptor, mammalian target of rapamycin inhibitors, and immuno-oncologic agents. ${ }^{1}$ Cytokine treatment was the standard of care prior to the introduction of molecular targeted agents, and during that era, a prognostic model was developed to predict survival in patients treated with interferon- $\alpha$ in clinical trials at the Memorial Sloan Kettering Cancer Center (MSKCC). ${ }^{2}$ The MSKCC model utilizes 5 clinical and laboratory pretreatment factors that independently predict overall survival (OS) to categorize patients into favorable-risk (0 risk factors), intermediate-risk (1-2 risk factors), or poor-risk ( $\geq 3$ risk factors) prognostic groups. ${ }^{2}$ Although developed during the cytokine era, the MSKCC prognostic model has since been validated for use with targeted agents. ${ }^{3}$ The International Metastatic Renal Cell Carcinoma Database Consortium (IMDC) model is another prognostic model developed from patients treated with firstline vascular endothelial growth factor-targeted therapy $(\mathrm{N}=645)$ and comprises 6 pretreatment clinical and laboratory factors. ${ }^{4}$ Similar to the MSKCC model, the IMDC model groups patients into favorable-risk ( 0 risk factors), intermediate-risk (1-2 risk factors), or poor-risk ( $\geq 3$ risk factors) groups, with significant differences in survival across risk groups. ${ }^{4}$ The MSKCC and IMDC risk classifications are widely used to estimate prognosis and are becoming increasingly important for selecting treatments for patients with advanced RCC, as evidenced by several new agents now available for patients with intermediate- or poor-risk RCC.,6

Even as the tyrosine kinase inhibitors pazopanib and sunitinib are standard first-line treatment options for patients with advanced RCC, ${ }^{7,8}$ the phase III CheckMate-214 study recently demonstrated superiority of nivolumab plus ipilimumab over sunitinib in patients with IMDC intermediate or poor risk. ${ }^{5}$ Conversely, patients with favorable risk in CheckMate-214 had significantly better outcomes (progression-free survival [PFS] and objective response rate [ORR]) with sunitinib compared with nivolumab plus ipilimumab in an exploratory analysis. ${ }^{5}$ Approximately one-half of patients with advanced RCC have MSKCC or IMDC intermediate risk, ${ }^{4,9-11}$ and discordant results between the favorable- and intermediate-risk populations in CheckMate- $214^{5}$ suggest that intermediate-risk patients may be further stratified to more accurately predict treatment outcomes. Indeed, previous retrospective analyses in intermediaterisk patients have demonstrated survival differences between patients with 1 versus 2 risk factors, ${ }^{10-12}$ supporting the heterogeneity of this patient subgroup and justifying further analyses in prospective datasets.

The large, prospective, observational PRINCIPAL study (NCT01649778) confirmed the efficacy and safety of pazopanib in a real-world clinical setting in 657 patients with advanced RCC. ${ }^{13}$ The objective of this secondary analysis of PRINCIPAL was to evaluate the real-world effectiveness and safety of pazopanib within MSKCC and IMDC intermediate-risk group patients with advanced RCC.

\section{Patients and Methods \\ Study Design and Patients}

PRINCIPAL was a global, prospective, observational study of patients with advanced/metastatic RCC treated with frontline pazopanib. The study was designed to enroll $\sim 500$ to 700 patients over approximately 30 months. This sample size was chosen based on the expected precision for the outcomes of interest $(<5 \%$ for PFS, OS, and ORR) and the feasibility of enrolling the desired patient population over the enrollment period. Consecutive patients meeting eligibility criteria were enrolled at participating sites and followed for 30 months or until premature discontinuation owing to death, withdrawal of consent, loss to follow-up, or termination from study. Patients who permanently discontinued study treatment were followed for up to 30 months post-enrollment. Patients were considered to have completed the study if 30 months of follow-up were conducted, or if the patient died during the study treatment or follow-up period.

Patients aged $\geq 18$ years who have advanced/metastatic RCC of clear-cell or predominantly clear-cell histology and who made a clinical decision to initiate pazopanib within 30 days of enrollment were eligible for participation in the PRINCIPAL study. These secondary analyses included only patients who met MSKCC or IMDC intermediate-risk criteria. Patients were classified as MSKCC intermediate risk if they had 1 or 2 of the following risk factors ${ }^{2}$ : time from initial diagnosis to initiation of therapy $<1$ year; Karnofsky performance status (KPS) $<80 \%$; serum hemoglobin level $<$ lower limit of normal (LLN); serum corrected calcium level $>10 \mathrm{mg} / \mathrm{dL}$; and lactate dehydrogenase level $>1.5 \times$ upper limit of

Table 1 Intermediate-risk Patient Disposition

\begin{tabular}{l|c|c} 
& MSKCC Intermediate Risk & $\begin{array}{c}\text { IMDC Intermediate Risk } \\
\mathbf{n = 3 4 3} \text { (\%) }\end{array}$ \\
Patients who completed study & $\mathbf{n = 3 6 3 ( \% )}$ & $258(75.2)$ \\
\hline Completed 30 months follow-up & $281(77.4)$ & $130(37.9)$ \\
\hline Deaths & $133(36.6)$ & $128(37.3)$ \\
\hline Patients who discontinued study & $148(40.8)$ & $85(24.8)$ \\
\hline Withdrawal of patient consent & $82(22.6)$ & $32(9.3)$ \\
\hline Physician request & $31(8.5)$ & $11(3.2)$ \\
\hline Lost to follow-up & $8(2.2)$ & $27(7.9)$ \\
\hline Other & $29(8.0)$ & $15(4.4)$ \\
\hline
\end{tabular}

Abbreviations: IMDC = International Metastatic Renal Cell Carcinoma Database Consortium; MSKCC = Memorial Sloan Kettering Cancer Center. 


\section{Pazopanib in Patients With Intermediate-risk Advanced RCC}

Table 2 Intermediate-risk Patient Baseline Demographics and Disease Characteristics

\begin{tabular}{|c|c|c|}
\hline & $\begin{array}{l}\text { MSKCC Intermediate Risk } \\
\qquad \mathbf{n}=363(\%)\end{array}$ & $\begin{array}{l}\text { IMDC Intermediate Risk } \\
n=343(\%)\end{array}$ \\
\hline Median age, y (range) & $67.0(22.0-89.0)$ & $67.0(22.0-90.0)$ \\
\hline Male & $256(70.5)$ & $243(70.8)$ \\
\hline \multicolumn{3}{|l|}{$\operatorname{Race}^{\mathrm{a}}$} \\
\hline White/Caucasian/European & $347(95.6)$ & $323(94.2)$ \\
\hline Other & $19(5.2)$ & $19(5.5)$ \\
\hline Unknown/declined to provide & 0 & $2(0.6)$ \\
\hline \multicolumn{3}{|l|}{ Number of risk factors ${ }^{\mathrm{b}}$} \\
\hline 1 & $147(40.5)$ & $171(49.9)$ \\
\hline 2 & $141(38.8)$ & $133(38.8)$ \\
\hline Missing & $75(20.7)$ & $39(11.4)$ \\
\hline \multicolumn{3}{|l|}{ ECOG performance status } \\
\hline$<2$ & $333(91.7)$ & $316(92.1)$ \\
\hline$\geq 2$ & $8(2.2)$ & $8(2.3)$ \\
\hline Not recorded & $22(6.1)$ & $19(5.5)$ \\
\hline Median disease duration of RCC from initial diagnosis, y (range) & $1.8(0.0-27.9)$ & $1.8(0.0-27.9)$ \\
\hline Median disease duration of locally advanced/metastatic RCC, y (range) & $0.1(0.0-23.1)$ & $0.1(0.0-23.1)$ \\
\hline Metastases present & $349(96.1)$ & $329(95.9)$ \\
\hline Median number of metastatic sites (range) & $2.0(0.0-15.0)$ & $2.0(0.0-15.0)$ \\
\hline \multicolumn{3}{|l|}{ Location of metastatic sites } \\
\hline Lung & $241(66.4)$ & $225(65.6)$ \\
\hline Lymph nodes & $117(32.2)$ & $112(32.7)$ \\
\hline Bone & $93(25.6)$ & $88(25.7)$ \\
\hline Liver & $53(14.6)$ & $45(13.1)$ \\
\hline Adrenal glands & $43(11.8)$ & $39(11.4)$ \\
\hline Brain & $16(4.4)$ & $14(4.1)$ \\
\hline Other & $91(25.1)$ & $88(25.7)$ \\
\hline Prior nephrectomy & $287(79.1)$ & $273(79.6)$ \\
\hline First-line systemic therapy & $18(5.0)$ & $14(4.1)$ \\
\hline Interleukin-2 & $3(0.8)$ & $1(0.3)$ \\
\hline Interferon- $\alpha$ & $8(2.2)$ & $6(1.7)$ \\
\hline Other & $7(1.9)$ & $7(2.0)$ \\
\hline \multicolumn{3}{|l|}{ Adjuvant/neoadjuvant systemic therapy } \\
\hline Adjuvant & $2(0.6)$ & $3(0.9)$ \\
\hline Neoadjuvant & $2(0.6)$ & $2(0.6)$ \\
\hline No & $328(90.4)$ & $313(91.3)$ \\
\hline Not applicable & $31(8.5)$ & $25(7.3)$ \\
\hline
\end{tabular}

Abbreviations: $E C O G=$ Eastern Cooperative Oncology Group; IMDC = International Metastatic Renal Cell Carcinoma Database Consortium; MSKCC = Memorial Sloan Kettering Cancer Center; $\mathrm{RCC}=$ renal cell carcinoma.

aPatients may have indicated more than one race category.

${ }^{\mathrm{b} P a t i e n t s ~ w i t h ~ o n e ~ m i s s i n g ~ r i s k ~ f a c t o r ~ w e r e ~ e x c l u d e d . ~}$

normal (ULN). Patients were classified as IMDC intermediate risk if they had 1 or 2 of the following risk factors ${ }^{4}$ : time from initial diagnosis to initiation of therapy $<1$ year; KPS $<80 \%$; serum hemoglobin level $<$ LLN; serum corrected calcium level $>$ ULN; absolute neutrophil count > ULN; and platelet count > ULN. Missing KPS risk factor was imputed based on baseline Eastern Cooperative Oncology Group (ECOG) performance status (PS) score: if baseline ECOG PS $<2$, then KPS risk factor $=$ no; if baseline ECOG PS $\geq 2$, then KPS risk factor $=$ yes.
All patients provided informed consent, and the study was conducted in accordance with International Conference on Harmonisation Good Clinical Practice, patient privacy requirements, and ethical principles outlined in the Declaration of Helsinki 2008.

\section{Assessments}

There were no visits or procedures mandated by the protocol. At the baseline visit, patient demographics, disease characteristics, and medical history were collected. Follow-up information was obtained 
Giuseppe Procopio et al

Table 3 Subgroup Analysis of PFS and OS in Patients With Intermediate Risk at Baseline

\begin{tabular}{|c|c|c|c|c|}
\hline & \multicolumn{2}{|c|}{ MSKCC Intermediate Risk } & \multicolumn{2}{|c|}{ IMDC Intermediate Risk } \\
\hline & $\begin{array}{c}\text { Disease Progression } \\
\text { or Death, } \mathbf{N}\end{array}$ & Median $(95 \% \mathrm{Cl})$, mos & $\begin{array}{l}\text { Disease Progression } \\
\text { or Death, N }\end{array}$ & Median (95\% Cl), mos \\
\hline \multicolumn{5}{|c|}{ Progression-free survival } \\
\hline \multicolumn{5}{|c|}{ Number of risk factors ${ }^{b}$} \\
\hline 1 & $85 / 147$ & $13.8(10.7-18.1)$ & $88 / 171$ & $13.1(10.7-18.1)$ \\
\hline 2 & $85 / 141$ & $7.4(6.2-10.3)$ & $88 / 133$ & $8.1(6.4-10.7)$ \\
\hline \multicolumn{5}{|l|}{ Age, y } \\
\hline$<65$ & $79 / 142$ & $12.3(9.0-16.4)$ & $73 / 136$ & $13.1(10.3-18.4)$ \\
\hline$\geq 65$ & $131 / 219$ & $10.7(9.0-13.8)$ & $123 / 205$ & $10.7(9.0-13.1)$ \\
\hline \multicolumn{5}{|c|}{ ECOG performance status } \\
\hline$<2$ & $189 / 333$ & $11.2(9.5-14.1)$ & $177 / 316$ & $11.8(9.9-15.4)$ \\
\hline$\geq 2$ & $8 / 8$ & $5.6(1.3-12.8)$ & $8 / 8$ & $2.3(1.2-10.7)$ \\
\hline \multicolumn{5}{|c|}{ Overall survival } \\
\hline \multicolumn{5}{|c|}{ Number of risk factors ${ }^{b}$} \\
\hline 1 & $46 / 147$ & NR (NE-NE) & $51 / 171$ & 33.9 (33.9-NE) \\
\hline 2 & $77 / 141$ & $15.2(12.3-26.5)$ & $66 / 133$ & 19.4 (14.3-NE) \\
\hline \multicolumn{5}{|l|}{ Age, y } \\
\hline$<65$ & $52 / 142$ & $33.9(27.0-33.9)$ & $43 / 136$ & 33.9 (NE-NE) \\
\hline$\geq 65$ & $95 / 219$ & 30.5 (19.9-NE) & $84 / 205$ & 32.9 (26.0-NE) \\
\hline \multicolumn{5}{|c|}{ ECOG performance status } \\
\hline$<2$ & $132 / 333$ & 33.9 (27.9-NE) & $113 / 316$ & 33.9 (30.5-NE) \\
\hline$\geq 2$ & $6 / 8$ & 9.5 (1.3-NE) & $7 / 8$ & $5.0(1.2-12.8)$ \\
\hline
\end{tabular}

Abbreviations: $\mathrm{Cl}=$ confidence interval; $\mathrm{ECOG}=$ Eastern Cooperative Oncology Group; IMDC = International Metastatic Renal Cell Carcinoma Database Consortium; MSKCC = Memorial Sloan Kettering Cancer Center; $\mathrm{NE}=$ not evaluable; $\mathrm{NR}=$ not reached.

${ }^{a}$ Analysis conducted in all treated (AT) population.

bPatients with 1 missing risk factor excluded.

approximately every 3 months ( \pm 4 weeks). Participating physicians assessed tumor responses according to local processes and their own clinical judgement. Primary effectiveness measures were PFS, OS, and ORR (defined as complete response or partial response).
Patients who received $\geq 1$ dose of pazopanib were evaluable for PFS, OS, and safety analyses (all treated [AT] population). The measurable disease (MD) population comprised patients with measurable disease at baseline and was used for the ORR analysis.

\section{Table 4 Subgroup Analysis of ORR in Patients With Intermediate Risk at Baseline}

\begin{tabular}{|c|c|c|c|c|c|c|}
\hline & \multicolumn{3}{|c|}{ MSKCC Intermediate Risk } & \multicolumn{3}{|c|}{ IMDC Intermediate Risk } \\
\hline & ORR, n/N (\%) & $\begin{array}{c}\text { Median DOR, mos } \\
(95 \% \mathrm{Cl})\end{array}$ & \begin{tabular}{|} 
Median TTR, mos \\
(95\% Cl)
\end{tabular} & ORR, n/N (\%) & $\begin{array}{c}\text { Median DOR, mos } \\
(95 \% \mathrm{Cl})\end{array}$ & $\begin{array}{c}\text { Median TTR, mos } \\
(95 \% \mathrm{Cl})\end{array}$ \\
\hline \multicolumn{7}{|c|}{$\begin{array}{l}\text { Number of risk } \\
\text { factors }^{b}\end{array}$} \\
\hline 1 & 42/124 (33.9) & $15(10.8-22.5)$ & $3(2.9-3.5)$ & 44/143 (30.8) & $14(7.5-22.5)$ & $3(2.9-3.2)$ \\
\hline 2 & $44 / 129(34.1)$ & $7(4.6-21.3)$ & $3(2.7-3.2)$ & $39 / 119(32.8)$ & $7(4.4-20.1)$ & $3(2.7-3.2)$ \\
\hline \multicolumn{7}{|l|}{ Age, y } \\
\hline$<65$ & 40/123 (32.5) & 14 (5.8-19.3) & $3(2.7-3.4)$ & 43/117 (36.8) & 15 (5.8-19.3) & 3 (2.9-3.5) \\
\hline$\geq 65$ & $62 / 190(32.6)$ & $14(8.8-22.3)$ & $3(2.8-3.1)$ & $53 / 178(29.8)$ & $11(7.1-20.1)$ & $3(2.8-3.1)$ \\
\hline \multicolumn{7}{|c|}{$\begin{array}{l}\text { ECOG performance } \\
\text { status }\end{array}$} \\
\hline$<2$ & 97/288 (33.7) & $14(7.2-19.1)$ & $3(2.9-3.1)$ & 93/272 (34.2) & $13(7.2-17.1)$ & $3(2.9-3.1)$ \\
\hline$\geq 2$ & $0 / 7(0.0)$ & NA & NA & $0 / 7(0.0)$ & NA & NA \\
\hline
\end{tabular}

Abbreviations: $\mathrm{DOR}=$ duration of response; $\mathrm{ECOG}=$ Eastern Cooperative Oncology Group; MSKCC $=$ Memorial Sloan Kettering Cancer Center; NA = not applicable; ORR = objective response rate; TTR $=$ time to response.

${ }^{\mathrm{a} A n a l y s i s ~ c o n d u c t e d ~ i n ~ m e a s u r a b l e ~ d i s e a s e ~(M D) ~ p o p u l a t i o n . ~}$

bPatients with 1 missing risk factor excluded. 


\section{Pazopanib in Patients With Intermediate-risk Advanced RCC}

\begin{tabular}{|c|c|c|c|c|}
\hline & \multicolumn{2}{|c|}{$\begin{array}{l}\text { MSKCC Intermediate Risk } \\
\qquad \mathrm{n}=363(\%)\end{array}$} & \multicolumn{2}{|c|}{$\begin{array}{l}\text { IMDC Intermediate Risk } \\
n=343(\%)\end{array}$} \\
\hline & All-grade & Grade $\geq 3$ & All-grade & Grade $\geq 3$ \\
\hline Any $\mathrm{AE}$ & $276(76.0)$ & $158(43.5)$ & $263(76.7)$ & $154(44.9)$ \\
\hline Treatment-related & $227(62.5)$ & $109(30.0)$ & $218(63.6)$ & $110(32.1)$ \\
\hline AESIs $^{\mathrm{a}}$ & $231(63.6)$ & $98(27.0)$ & $224(65.3)$ & $99(28.9)$ \\
\hline Treatment-related & $210(57.9)$ & $86(23.7)$ & $203(59.2)$ & $89(25.9)$ \\
\hline SAES & $97(26.7)$ & $76(20.9)$ & $92(26.8)$ & $70(20.4)$ \\
\hline Treatment-related & 38 (10.5) & $31(8.5)$ & $34(9.9)$ & $28(8.2)$ \\
\hline Fatal SAEs & $17(4.7)$ & $17(4.7)$ & $13(3.8)$ & $13(3.8)$ \\
\hline Treatment-related & $1(0.3)$ & $1(0.3)$ & $1(0.3)$ & $1(0.3)$ \\
\hline AEs leading to treatment discontinuation & $53(14.6)$ & $29(8.0)$ & $54(15.7)$ & $29(8.5)$ \\
\hline Treatment-related & $39(10.7)$ & $21(5.8)$ & $43(12.5)$ & $24(7.0)$ \\
\hline AEs leading to dose adjustment/interruption & $190(52.3)$ & $104(28.7)$ & $186(54.2)$ & $101(29.4)$ \\
\hline
\end{tabular}

Abbreviations: AEs = adverse events; AESI = adverse events of special interest; IMDC = International Metastatic Renal Cell Carcinoma Database Consortium; MSKCC = Memorial Sloan Kettering Cancer Center; SAEs = serious adverse events.

${ }^{a}$ AESIs were defined as any reports of new onset/worsened hypertension, cardiac dysfunction, thyroid dysfunction, evidence of liver toxicity, or any other AE resulting in a pazopanib dose modification or discontinuation.

\section{Statistical Analyses}

Continuous variables were reported as medians and ranges; categorical variables were reported as number and percentage of the total population. Evaluations were based on point estimates and 95\% confidence intervals (CIs), and efficacy and safety analyses were stratified by baseline patient characteristics (eg, ECOG PS, histologic subtype). Formal hypothesis or statistical significance testing was not planned.

\section{Results \\ Patients}

Of the 657 enrolled patients in the PRINCIPAL study who received $\geq 1$ dose of pazopanib (AT population), more than onehalf had intermediate-risk criteria per MSKCC (55.3\%; $\mathrm{n}=363$ ) and IMDC $(52.2 \% ; \mathrm{n}=343)$; MSKCC and IMDC risk data were missing for $178(27.1 \%)$ and $128(19.5 \%)$ patients in the overall population, respectively. Among the MSKCC and IMDC intermediate-risk patients, $281(77.4 \%)$ and 258 (75.2\%) completed the study, and 133 (36.6\%) and 130 (37.9\%) completed the 30-month follow-up. One hundred and forty-eight (40.8\%) in the MSKCC intermediate-risk group and 128 (37.3\%) in the IMDC intermediate-risk group died. Discontinuation rates were similar for both intermediate-risk groups (Table 1). Baseline patient demographics and disease characteristics for intermediate-risk patients are shown in Table 2. Per MSKCC and IMDC criteria, 1 risk factor was present for 147 (40.5\%) and 171 (49.9\%) patients, respectively, and 2 risk factors were present for 141 (38.8\%) and $133(38.8 \%)$ patients, respectively. Most patients had an ECOG PS of $<2$.

\section{Effectiveness}

In both MSKCC and IMDC intermediate-risk groups, median PFS and OS were numerically longer for patients who had 1 risk factor compared with patients who had 2 risk factors. Median PFS was 13.8 months (95\% CI, 10.7-18.1 months) and 7.4 months (95\% CI, 6.2-10.3 months) for patients with 1 and 2 MSKCC risk factors, respectively, and 13.1 months (95\% CI, 10.7-18.1 months) and 8.1 months (95\% CI, 6.4-10.7 months) for patients with 1 and 2 IMDC risk factors, respectively (Table 3). Within these intermediate-risk populations, the median OS was not reached and was 15.2 months (95\% CI, 12.3-26.5 months) for patients with 1 and 2 MSKCC risk factors, respectively, and 33.9 months (95\% CI, 33.9 months to not estimable) and 19.4 months (95\% CI, 14.3 months to not estimable) for patients with 1 and 2 IMDC risk factors, respectively (Table 3). Shorter median PFS and OS were observed in patients with ECOG PS $\geq 2$ (vs. PS $<2$ ) (Table 3 ).

Among patients with intermediate-risk, a lower ORR was observed for those with ECOG PS $\geq 2$ (vs. PS $<2$ ). For patients with ECOG PS $<2$, the ORR was $33.7 \%$ and $34.2 \%$ by MSKCC and IMDC intermediate-risk criteria, respectively, compared with $0 \%$ for patients with ECOG PS $\geq 2$ by both MSKCC and IMDC intermediate-risk criteria (Table 4).

\section{Table 6 Treatment-related Adverse Events}

\begin{tabular}{|c|c|c|}
\hline $\begin{array}{l}\text { Any Grade } \\
\text { Occurring in } \\
\geq 5 \% \text { of Patients } \\
\text { in Either Group }\end{array}$ & $\begin{array}{c}\text { MSKCC } \\
\text { Intermediate Risk } \\
\mathbf{n}=363(\%)\end{array}$ & $\begin{array}{c}\text { IMDC } \\
\text { Intermediate Risk } \\
\mathbf{n}=\mathbf{3 4 3}(\%)\end{array}$ \\
\hline Hypertension & $86(23.7)$ & $82(23.9)$ \\
\hline Diarrhea & 45 (12.4) & $47(13.7)$ \\
\hline ALT increased & 45 (12.4) & 42 (12.2) \\
\hline AST increased & $28(7.7)$ & $26(7.6)$ \\
\hline Hypothyroidism & $21(5.8)$ & $24(7.0)$ \\
\hline Nausea & $19(5.2)$ & $16(4.7)$ \\
\hline Blood TSH increased & $15(4.1)$ & $18(5.2)$ \\
\hline
\end{tabular}

Abbreviations: ALT = alanine aminotransferase; AST = aspartate aminotransferase; $\mathrm{TSH}=$ thyroid-stimulating hormone. 


\begin{tabular}{|c|c|c|c|c|}
\hline \multirow{2}{*}{$\begin{array}{l}\text { Any Grade Occurring } \\
\text { in } \geq 5 \% \text { of Patients } \\
\text { in any Group }\end{array}$} & \multicolumn{2}{|c|}{$\begin{array}{l}\text { MSKCC Intermediate Risk } \\
\qquad \mathbf{n}=363(\%)\end{array}$} & \multicolumn{2}{|c|}{$\begin{array}{l}\text { IMDC Intermediate Risk } \\
n=343(\%)\end{array}$} \\
\hline & $\begin{array}{c}<65 \text { Years } \\
n=143\end{array}$ & $\begin{array}{c}\geq 65 \text { Years } \\
n=220\end{array}$ & $\begin{array}{c}<65 \text { Years } \\
n=137\end{array}$ & $\begin{array}{c}\geq 65 \text { Years } \\
n=206\end{array}$ \\
\hline Hypertension & 38 (26.6) & $52(23.6)$ & $41(29.9)$ & $47(22.8)$ \\
\hline ALT increased & $21(14.7)$ & $25(11.4)$ & $19(13.9)$ & $24(11.7)$ \\
\hline Diarrhea & 18 (12.6) & 30 (13.6) & $23(16.8)$ & 27 (13.1) \\
\hline AST increased & $15(10.5)$ & $15(6.8)$ & $14(10.2)$ & $13(6.3)$ \\
\hline Blood TSH increased & $13(9.1)$ & $9(4.1)$ & $16(11.7)$ & $9(4.4)$ \\
\hline Nausea & $11(7.7)$ & $11(5.0)$ & $10(7.3)$ & $9(4.4)$ \\
\hline Vomiting & $9(6.3)$ & $6(2.7)$ & $7(5.1)$ & $4(1.9)$ \\
\hline Hypothyroidism & $8(5.6)$ & $14(6.4)$ & $9(6.6)$ & $17(8.3)$ \\
\hline Asthenia & $5(3.5)$ & $11(5.0)$ & $5(3.6)$ & $9(4.4)$ \\
\hline Fatigue & $3(2.1)$ & $11(5.0)$ & $2(1.5)$ & $10(4.9)$ \\
\hline Hepatotoxicity & $2(1.4)$ & $12(5.5)$ & $3(2.2)$ & $13(6.3)$ \\
\hline
\end{tabular}

Abbreviations: ALT = alanine aminotransferase; AST = aspartate aminotransferase; TSH $=$ thyroid-stimulating hormone.

\section{Safety}

At least 1 adverse event (AE) was reported by most patients with intermediate risk (MSKCC, 76.0\%; IMDC, 76.7\%), and most AEs were related to treatment (MSKCC, 62.5\%; IMDC, 63.6\%) (Table 5). The most commonly reported treatment-related AEs $(\geq 5 \%)$ in the MSKCC and IMDC intermediate-risk groups were hypertension, diarrhea, increased alanine aminotransferase, increased aspartate aminotransferase, hypothyroidism, nausea, and increased blood thyroid-stimulating hormone (Table 6). Treatmentrelated serious AEs were reported by 38 (10.5\%) MSKCC intermediate-risk patients and 34 (9.9\%) IMDC intermediate-risk patients. Seventeen $(4.7 \%)$ and $13(3.8 \%)$ patients died in the MSKCC and IMDC intermediate-risk groups, respectively; 1 $(0.3 \%)$ death in each intermediate-risk group was considered related to treatment. When pazopanib safety was assessed in intermediaterisk patients aged $\geq 65$ and $<65$ years, the $\mathrm{AE}$ profile was generally similar between the 2 age groups (Table 7 ).

\section{Discussion}

Prognostic risk models based on clinical and laboratory factors not only predict survival for patients with advanced RCC but also have become important tools to guide treatment choice in a crowded therapeutic landscape. New treatment options have recently emerged for intermediate- or poor-risk patients ${ }^{5,6}$; however, it is unclear whether all intermediate-risk patients, who comprise a heterogeneous patient group with 1 or 2 risk factors, should be treated similarly. Such differential efficacy outcomes between risk groups have been previously demonstrated in the CheckMate-214 trial, for example. ${ }^{5}$ In CheckMate-214, nivolumab plus ipilimumab showed superior OS and ORR versus sunitinib in intermediate-/poor-risk patients (co-primary endpoints). Conversely, PFS and ORR favored sunitinib in an exploratory analysis of favorable-risk patients $^{5}$; however, the difference in ORR between treatment groups was no longer significant in an updated analysis after a minimum of 30 months' follow-up (updated PFS not reported). ${ }^{14}$

The current secondary analysis of the PRINCIPAL study investigates intermediate-risk subgroups for differences in outcomes. An earlier primary analysis of the PRINCIPAL study demonstrated that the PFS of the overall MSKCC and IMDC intermediate-risk patients was 11.2 months (95\% CI, 9.5-13.7 months) and 11.6 months (95\% CI, 9.8-14.1 months), respectively; OS was 33.9 months (95\% CI, 26.9 months to not reached) and 32.9 months (95\% CI, 28.6 months to not reached), respectively. In this large prospective real-world study, intermediate-risk patients with 1 risk factor by both MSKCC and IMDC criteria had longer median PFS and OS with pazopanib compared with patients with 2 risk factors. Clinical outcomes were also worse for patients with poor ECOG PS $(\geq 2)$ versus patients with ECOG PS $<2$. These results highlight the heterogeneity of the intermediate-risk group. Further, our findings are consistent with previous retrospective analyses of intermediate-risk advanced RCC patients undergoing targeted therapy, which found prolonged PFS and $O S$ in patients with 1 risk factor compared with 2 risk factors ${ }^{10-12}$ and in patients with poorer ECOG PS (0 vs. 1-2). ${ }^{11}$ Findings from the secondary analysis of the PRINCIPAL study suggest that patients with advanced RCC of intermediate prognostic risk can be further stratified, by 1 risk factor (intermediate-low) versus 2 risk factors (intermediate-high) or ECOG PS to more accurately predict treatment outcomes. These findings may further aid treatment choice for patients classified as having intermediate risk, particularly if applied to clinical trials of immuno-oncologic regimens versus tyrosine kinase inhibitor therapy.

Pazopanib treatment among intermediate-risk patients was generally well-tolerated. A relatively low frequency of hypertension, diarrhea, alanine aminotransferase elevations, and aspartate aminotransferase elevations were observed compared with past clinical trial data, possibly owing to the observational nature of PRINCIPAL. Further, we noted similar safety profiles for patients in the older ( $\geq 65$ years) and younger ( $<65$ years) age groups, which supports a role for pazopanib in the treatment of patients of all ages with advanced RCC.

\section{Conclusions}

Patients with intermediate-risk advanced RCC treated with pazopanib in the prospective observational PRINCIPAL study 



\section{Giuseppe Procopio et al}

intermediate-risk group of the Memorial Sloan Kettering Cancer Center criteria. Oncotarget 2018; 9:27752-9.

11. Sella A, Michaelson MD, Matczak E, Simantov R, Lin X, Figlin RA. Heterogeneity of patients with intermediate-prognosis metastatic renal cell carcinom treated with sunitinib. Clin Genitourin Cancer 2017; 15:291-9.e1.

12. Iacovelli R, De Giorgi U, Galli L, et al. Is it possible to improve prognostic classification in patients affected by metastatic renal cell carcinoma with an intermediate or poor prognosis? Clin Genitourin Cancer 2018; 16:355-9.e1.
13. Schmidinger M, Bamias A, Procopio G, et al. Prospective observational study of pazopanib in patients with advanced renal cell carcinoma (PRINCIPAL Study). Oncologist 2019. https://doi.org/10.1634/theoncologist. 2018-0787.

14. Rini BI, Tannir NM, Escudier B, et al. Characterization of response to nivolumab plus ipilimumab $(\mathrm{N}+\mathrm{I})$ or sunitinib $(\mathrm{S})$ in patients (Pts) with previously untreated advanced renal cell carcinoma (aRCC): Checkmate 214. Ann Oncol 2018; 29(8 Suppl), Abstract 875P. 